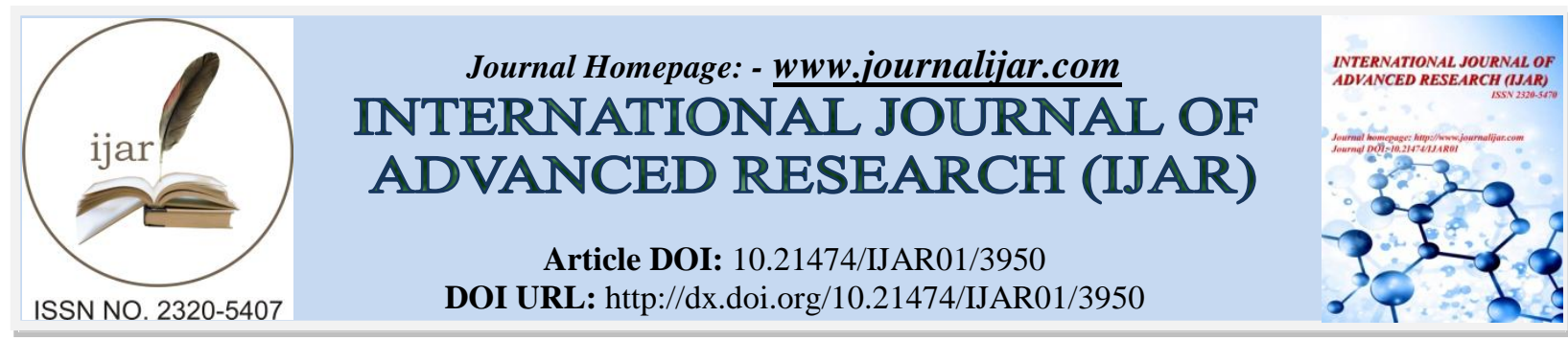

RESEARCH ARTICLE

\title{
DEVELOPMENT OF MODULATIONAL INSTABILITIES IN TRAVELING WAVE SOLUTIONS FOR WAVE-WAVE INTERACTION IN IONIC MEDIA.
}

\author{
Kavita Sharma ${ }^{1}$, Arun Kumar ${ }^{2}$ and Ram Dayal Pankaj ${ }^{3}$. \\ 1. Research Scholar, Department of Mathematics, Career Point University, Kota (Raj.), India. \\ 2. Department of Mathematics, Government College Kota, Kota (Raj.), India. \\ 3. Department of Mathematics, J.N.V. University, Kota (Raj.), India.
}

\section{Manuscript Info \\ Manuscript History}

Received: 12 February 2017

Final Accepted: 16 March 2017

Published: April 2017

\begin{abstract}
In this paper, the development of modulational instabilities are shown in traveling wave solutions which are calculated using exp $(-\phi(\xi))$ expansion Method. These solutions completely new and have not found in earlier. But the solutions found in the Ref. [20] are the same to our obtain solutions. By means of this scheme, we found some new travelling wave solutions of the above mentioned equations. The $\exp (-$ $\Phi(\xi))$ - expansion method can be easily applied to solve the NLDEs and provides some new solutions. The solutions obtained in this article have been checked by putting them back into the original equation and found correct.
\end{abstract}

Copy Right, IJAR, 2017,. All rights reserved.

\begin{abstract}
Introduction:-
It is now accepted in the scientific community that large amplitude waves can appear on the surface of the ocean; whether these events are caused by a "fortuitous" linear superposition of different waves with the same phases or are the result of weakly nonlinear interactions is still a debated issue. For linear waves the fundamental work was done by Rice [1] in connection with noise in electronic circuits. Some years later Longuet-Higgins [2] adapted the ideas of Rice to surface gravity waves. He showed that if the wave spectrum is narrow banded and if the phases of the Fourier components of the surface elevation are distributed uniformly (random phases), then the probability distribution of wave heights, crests and troughs is given by the Rayleigh distribution. Corrections to this distribution for wave crests can be obtained if for each wave (free mode), its bound contribution is included. For the narrowband case this is nothing but describing the surface elevation as a Stokes expansion. The general description of the surface elevation that takes into account bound modes up to second order in wave steepness was given in a seminal paper by Longuet Higgins [3]. The numerical implementation of the formulas reported in the paper by LonguetHiggins corresponds to what today is called the "second order theory". In the narrow-band approximation, for infinite water depth and under the hypothesis that free waves are described by a Gaussian statistics, Tayfun [4] derived a formula for the distribution of wave crests (now known as the Tayfun distribution) which enhances the tail of the Rayleigh distribution, especially if the wave steepness is large. It should be stressed that the Tayfun second order theory still predicts a Rayleigh distribution for wave heights (this is because second order contributions cancel out for wave heights).
\end{abstract}

Corresponding Author:- Arun Kumar.

Address:- Department of Mathematics, Government College Kota, Kota (Raj.), India. 
Nonlinearity is a fascinating element of nature, today, many scientists see nonlinear science as the most important frontier for the fundamental understanding of nature. Many complex physical phenomena are frequently described and modelled by nonlinear evolution equation, so the exact or analytical solutions of the discussed nonlinear evolution equation become more and more important, which is considered not only a valuable tool in checking the accuracy of computational dynamics, but also gives us a good help to readily understand the essentials of complex physical phenomenon, e.g., collision of two solitary solutions. Looking for exact solitary wave solutions to nonlinear evolution equations has long been a major concern for both mathematicians and physicists. These solutions may well describe various phenomena in physics and other fields, such as solitons and propagation with a finite speed, and thus they may give more insight into the physical aspects of the problems. Modern theories of nonlinear science have been highly developed over the last half century.

At the classical level, a set of coupled nonlinear wave equations describing the interaction between high-frequency Langmuir waves and low-frequency ion-acoustic waves were firstly derived by Zakharov [5]. Since then, this system has been the subject of a large number of studies. In one dimension, the Zakharov Equations (ZE) may be written as

$$
\begin{aligned}
& i E_{t}+E_{x x}-n E=0, \\
& n_{t t}-n_{x x}-|E|_{x x}{ }^{2}=0
\end{aligned}
$$

Where $\mathrm{E}$ is the envelope of the high-frequency electric field, $n$ is the plasma density measured from its equilibrium value. The system can be derived from a hydrodynamic description of the plasma [6, 7]. However, some important effects such as transit-time damping and ion nonlinearities, which are also implied by the fact that the values used for the ion damping have been anomalously large from the point of view of linear ion-acoustic wave dynamics, have been ignored in the Zakharov Equations. This is equivalent to say that, the Zakharov Equations is a simplified model of strong Langmuir turbulence. Thus we have to generalize the Zakharov Equations by taking more elements into account. Starting from the dynamical plasma equations with the help of relaxed Zakharov simplification assumptions, and through taking use of the time-averaged two-time-scale two-fluid plasma description, the ZE are generalized to contain the self-generated magnetic field [8]. The generalized Zakharov equations (GZE) are a set of coupled equations and may be written as [9]

$$
\begin{aligned}
& i E_{t}+E_{x x}-2 \beta|E|^{2} E+2 n E=0, \\
& n_{t t}-n_{x x}+|E|_{x x}{ }^{2}=0
\end{aligned}
$$

where $\mathrm{E}$ is the envelope of the high-frequency electric field, and $n$ is the plasma density measured from its equilibrium value. This system is reduced to the classical Zakharov equations of plasma physics whenever $\beta=0$. Due to the fact that the GZE is a realistic model in plasma, it makes sense to study the solitary wave solutions of the GZE. Recently various powerful mathematical methods such as homotopy perturbation method [10], variational iteration method [11-18], Adomian decomposition method [19] and others [20-24] have been proposed to obtain exact and approximate analytic solutions for nonlinear problems.

\section{Description of exp $(-\phi(\xi))$-expansion Method:-}

We now present briefly the main steps of the exp $(-\phi(\xi))$-expansion strategy that will be applied. A PDE

$$
F\left(u, u_{t}, u_{x}, u_{y}, u_{t t}, u_{x x}, u_{y y}, u_{t x} \ldots\right)=0
$$

Where $u=u(x, t)$ is an unknown function, $F$ is a polynomial of $u(x, t)$ and its partial derivatives in which the highest order derivatives and nonlinear terms are involved.

Step-1 Using a wave variable $\xi=x+y \pm c t, \mathrm{c}$ is the speed of the traveling wave the traveling wave transformation $\xi=x+y \pm c t$ eq. (3) can be converted to an ODE for

$$
P\left(u, u^{\prime}, u^{\prime \prime}, u^{\prime \prime \prime} \ldots . .\right)=0
$$$$
u=u(\xi)
$$

Where, $P$ is a polynomial of $\mathrm{u}$ and its derivatives and the superscripts indicate the ordinary derivatives with respect to $\xi$. 
Step-2 Suppose the traveling wave solution of Equation (4) can be expressed as follows

$$
u(\xi)=\sum_{i=0}^{m} A_{i}\{\exp (-\Phi(\xi))\}^{i}
$$

Where $A_{i}$ are constants to be determined such that $A_{m} \neq 0 \quad(1 \leq i \leq m)$ and $\Phi=\Phi(\xi)$ satisfied the following differential equations

$$
\Phi^{\prime}(\xi)=\exp \{-\Phi(\xi)\}+\mu \exp \{-\Phi(\xi)\}+\lambda
$$

Eq. (6) gives the following solutions

Solution $1: \mu \neq 0,\left(\lambda^{2}-4 \mu\right)>0$

$$
\Phi(\xi)=\operatorname{In}\left(\frac{-\sqrt{\left(\lambda^{2}-4 \mu\right)} \tanh \left(\frac{\sqrt{\left(\lambda^{2}-4 \mu\right)}}{2}(\xi+\hbar)\right)-\lambda}{2 \mu}\right)
$$

Solution $2: \mu \neq 0,\left(\lambda^{2}-4 \mu\right)<0$

$$
\Phi(\xi)=\operatorname{In}\left(\frac{\sqrt{\left(4 \mu-\lambda^{2}\right)} \tan \left(\frac{\sqrt{\left(4 \mu-\lambda^{2}\right)}}{2}(\xi+\hbar)\right)-\lambda}{2 \mu}\right)
$$

Solution $3: \mu=0, \lambda \neq 0$, and $\left(\lambda^{2}-4 \mu\right)>0$

$$
\Phi(\xi)=-\operatorname{In}\left(\frac{\lambda}{\exp (\lambda(\xi+\hbar))-1}\right)
$$

Solution $4: \mu \neq 0, \lambda \neq 0$, and $\left(\lambda^{2}-4 \mu\right)=0$

$$
\Phi(\xi)=\operatorname{In}\left(-\frac{2(\lambda(\xi+\hbar)+2)}{\lambda^{2}(\xi+\hbar)}\right)
$$

Solution 5: $\mu=0, \lambda=0$, and $\left(\lambda^{2}-4 \mu\right)=0$

$$
\Phi(\xi)=\operatorname{In}(\xi+\hbar)
$$

where $\hbar, \lambda, \mu$ and $A_{m} \neq 0$ are an arbitrary constants to be determined later. The positive integer $m$ can be determined by considering the homogeneous balance between the highest order derivatives and the nonlinear terms appearing in Eq. (4).

Step-3 We substitute Eq. (5) into (4) and then we account the function exp (- $\Phi(\xi))$. As a result of this substitution, we get a polynomial of $\exp (-\Phi(\xi))$. We equate all the coefficients of same power of $\exp (-\Phi(\xi))$ to zero. This procedure yields a system of algebraic equations whichever can be solved to find, $\hbar, \lambda, \mu$ and $A_{0}, A_{1}, A_{0} \ldots$. with the aid of Maple. Substituting the values of $\hbar, \lambda, \mu$ and $A_{0}, A_{1}, A_{0} \ldots$ into Eq. (5) along with general solutions of Eq. (6) completes the determination of the solution of Eq. (2).

\section{Application exp $(-\phi(\xi))$-expansion Method for Generalized Zakharov Equations:-}

We introduce a transformation for (GZE) eq. (2)

$$
E(x, t)=U(\xi) e^{i \theta}, \quad \eta(x, t)=V(\xi), \quad \theta=k x+\omega t, \quad \xi=p(x-2 k t)
$$


where $\mathrm{k}, \omega$ and $\mathrm{p}$ are real constant. Put these transformation in equation (2), we have the ordinary differential equation (ODE) for $U(\xi)$ and $V(\xi)$

$$
\begin{aligned}
& U(\xi)\left(k^{2}+\omega\right)-p^{2} U^{\prime \prime}(\xi)+2 \beta U^{3}(\xi)-2 U(\xi) V(\xi)=0, \\
& \left(4 k^{2}-1\right) V^{\prime \prime}(\xi)+U^{\prime \prime}(\xi)=0
\end{aligned}
$$

where prime denotes the differential with respect to $\xi$.integration of second equation of eq.(12) twice with respect

to $\xi . \quad V(\xi)=\frac{C-U^{2}(\xi)}{\left(4 k^{2}-1\right)}$

where $\mathrm{C}$ is second integration constant and the first one is taken to zero. The value of $V(\xi)$ is put in first equation

$$
U(\xi)\left(k^{2}+\omega-\frac{2 C}{4 k^{2}-1}\right)-p^{2} U^{\prime \prime}(\xi)+2\left(\beta+\frac{1}{4 k^{2}-1}\right) U^{3}(\xi)=0
$$

Obtain after integrating the ODE once and setting the constant of integration equal to zero. Balancing $U^{\prime \prime}$ with $U^{3}$ in eq. (8) gives $m+2=3 m$ i.e. $m=1$

Therefore, the $\exp (-\phi(\xi))$-expansion Method allow us to use of the finite expansion

$$
U(\xi)=a_{0}+a_{1} \exp \left(-\phi(\xi), \quad a_{1} \neq 0\right.
$$

Substituting equation (15) into equation (14) and equating all terms with the powers in $(\exp (-\phi(\xi)))^{i}$, and setting each of the obtained coefficients for $(\exp (-\phi(\xi)))^{i}(i=0,1,2,3,4 \ldots)$ to zero, yields the set of algebraic equations for $\lambda, \mu, p, k, \omega$ and $\beta$, we obtain

$$
\begin{gathered}
\lambda= \pm \frac{2 a_{0}}{p} \sqrt{\frac{\beta\left(4 k^{2}-1\right)+1}{\left(4 k^{2}-1\right)}} \\
\mu=\frac{2 a_{0}\left\lfloor\beta\left(4 k^{2}-1\right)+1\right\rfloor-\left\lfloor 2 c-\left(k^{2}+\omega\right)\left(\left(4 k^{2}-1\right)\right)\right]}{2 p^{2}\left(4 k^{2}-1\right)} \\
a_{0}=a_{0} \quad a_{1}= \pm p \sqrt{\frac{\left(4 k^{2}-1\right)}{\beta\left(4 k^{2}-1\right)+1}}
\end{gathered}
$$

Where $p, c, k, \beta$ are arbitrary constants.

According to Equation (17), the travelling wave solutions of the Sch-Zakh Equation (2) with the help of Equation (13) and (15) are obtained in the following form:

Solution:-1 by equation (7), when $\mu \neq 0$,

$$
\Omega=\lambda^{2}-4 \mu=\frac{2\left[2 c-\left(k^{2}+\omega\right)\left(4 k^{2}-1\right)\right]}{p^{2}\left(4 k^{2}-1\right)}>0, \beta>0
$$

we have

$$
E_{1}(x, t)=e^{i(k x+\omega t)}\left[a_{0} \mp \frac{2 a_{0}\left[\beta\left(4 k^{2}-1\right)+1\right]-\left[2 c-\left(k^{2}+\omega\right)\left(\left(4 k^{2}-1\right)\right)\right]}{2 p^{2}\left(4 k^{2}-1\right) \sqrt{\Omega} \tanh \left[\frac{\sqrt{\Omega}}{2}(p(x-2 k t)+\hbar)\right] \pm \frac{2 a_{0}}{p} \sqrt{\frac{\beta\left(4 k^{2}-1\right)+1}{\left(4 k^{2}-1\right)}}}\right]
$$




$$
\eta_{1}(x, t)=\frac{c}{\left(4 k^{2}-1\right)}-\frac{1}{\left(4 k^{2}-1\right)}\left[a_{0} \mp \frac{2 a_{0}\left[\beta\left(4 k^{2}-1\right)+1\right]-\left[2 c-\left(k^{2}+\omega\right)\left(\left(4 k^{2}-1\right)\right)\right]}{2 p^{2}\left(4 k^{2}-1\right) \sqrt{\Omega} \tanh \left[\frac{\sqrt{\Omega}}{2}(p(x-2 k t)+\hbar)\right] \pm \frac{2 a_{0}}{p} \sqrt{\frac{\beta\left(4 k^{2}-1\right)+1}{\left(4 k^{2}-1\right)}}}\right]^{2}
$$

Solution:-2 When $\mu \neq 0, \Omega=\lambda^{2}-4 \mu=\frac{2\left[2 c-\left(k^{2}+\omega\right)\left(4 k^{2}-1\right)\right]}{p^{2}\left(4 k^{2}-1\right)}<0, \beta>0$ we obtain

$$
\begin{aligned}
& E_{2}(x, t)=e^{i(k x+\omega t)}\left[a_{0} \pm \frac{2 a_{0}\left[\beta\left(4 k^{2}-1\right)+1\right]-\left[2 c-\left(k^{2}+\omega\right)\left(\left(4 k^{2}-1\right)\right)\right]}{\left.2 p^{2}\left(4 k^{2}-1\right) \sqrt{-\Omega} \tan \left[\frac{\sqrt{-\Omega}}{2}(p(x-2 k t)+\hbar)\right] \mp \frac{2 a_{0}}{p} \sqrt{\frac{\beta\left(4 k^{2}-1\right)+1}{\left(4 k^{2}-1\right)}}\right]}\right. \\
& \eta_{2}(x, t)=\frac{c}{\left(4 k^{2}-1\right)}-\frac{1}{\left(4 k^{2}-1\right)}\left[a_{0} \pm \frac{2 a_{0}\left[\beta\left(4 k^{2}-1\right)+1\right]-\left[2 c-\left(k^{2}+\omega\right)\left(\left(4 k^{2}-1\right)\right)\right]}{2 p^{2}\left(4 k^{2}-1\right) \sqrt{\Omega} \tan \left[\frac{\sqrt{\Omega}}{2}(p(x-2 k t)+\hbar)\right] \mp \frac{2 a_{0}}{p} \sqrt{\frac{\beta\left(4 k^{2}-1\right)+1}{\left(4 k^{2}-1\right)}}}\right]^{2}
\end{aligned}
$$

Solution:-3 When $\mu=0, \lambda \neq 0,\left(\lambda^{2}-4 \mu\right)>0, \beta<0$

we obtain

$$
\begin{aligned}
& E_{3}(x, t)=e^{i(k x+\omega t)}\left[A_{0} \pm \frac{\frac{2 A_{0}}{P} \sqrt{\beta+\frac{1}{4 k^{2}-1}}}{\left.\exp \frac{2 A_{0}}{P} \sqrt{\beta+\frac{1}{4 k^{2}-1}}\{p(x-2 k t)+h\}-1\right]}\right]
\end{aligned}
$$

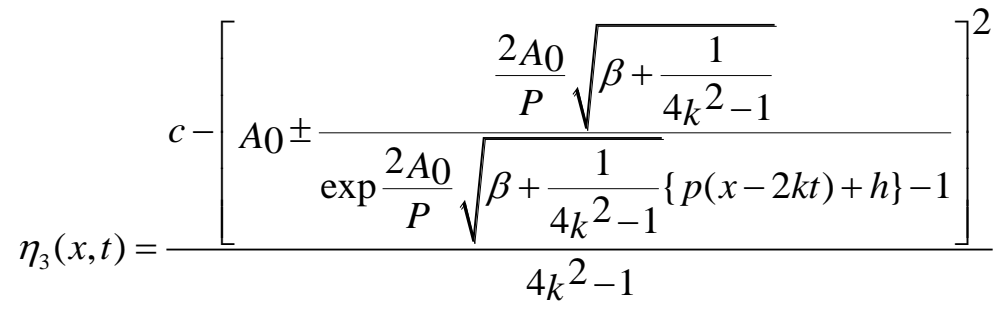

Solution:-4 When $\mu \neq 0, \lambda \neq 0,\left(\lambda^{2}-4 \mu\right)=0$, we obtain 


$$
\begin{aligned}
& E_{4}(x, t)=e^{i(k x+\omega t)}\left[A_{0} \mp \frac{\left.\frac{4 A_{0}^{2}}{p \sqrt{\beta+\frac{1}{4 k^{2}-1}}}\left(\beta+\frac{1}{4 k^{2}-1}\right)\{p(x-2 k t)+h\}\right]}{2\left[\frac{2 A 0}{P} \sqrt{\beta+\frac{1}{4 k^{2}-1}}\{p(x-2 k t)+h\}+2\right]}\right]
\end{aligned}
$$

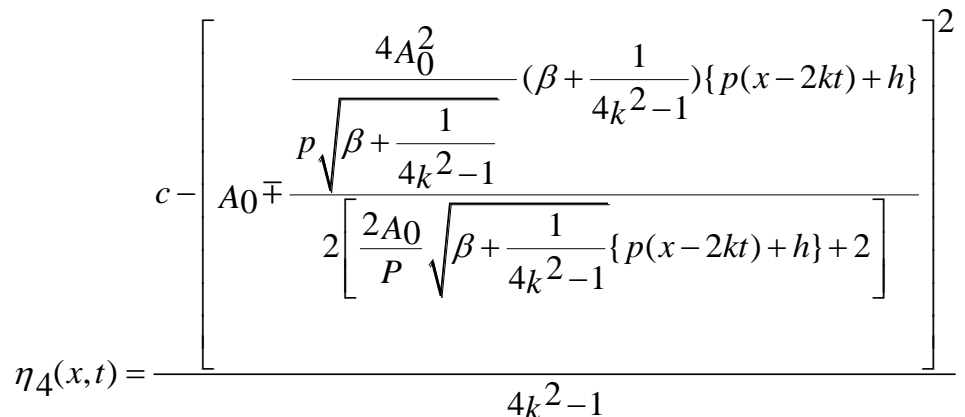

Solution:-5 When $\mu=0, \lambda=0,\left(\lambda^{2}-4 \mu\right)=0$, we obtain

$$
\begin{aligned}
& E_{5}(x, t)=e^{i(k x+\omega t)}\left[A_{0} \pm \frac{p}{[p(x-2 k t)+h] \sqrt{\beta+\frac{1}{4 k^{2}-1}}}\right] \\
& \eta_{5}(x, t)=\frac{c-\left[A_{0} \pm \frac{p}{[p(x-2 k t)+h] \sqrt{\beta+\frac{1}{4 k^{2}-1}}}\right.}{4 k^{2}-1}
\end{aligned}
$$

\section{Results and Discussion:-}

The explicit exact solutions of the coupled nonlinear Equation (2) play an important role for describing different types of wave propagation and of plasma as well as fluid mechanics. The exact traveling wave solutions are obtained from the explicit solutions by choosing the particular value of the physical parameters. So, we can choose appropriate value of the physical parameters to obtain exact solutions we need in varied instances.

(a)
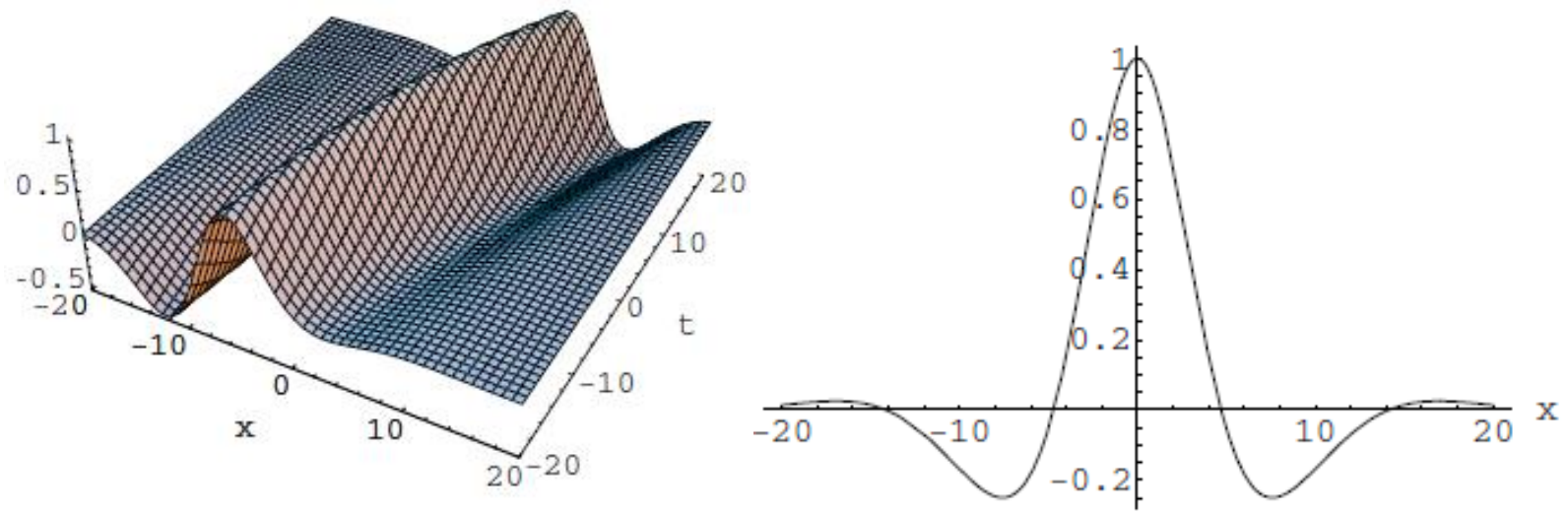
(b)
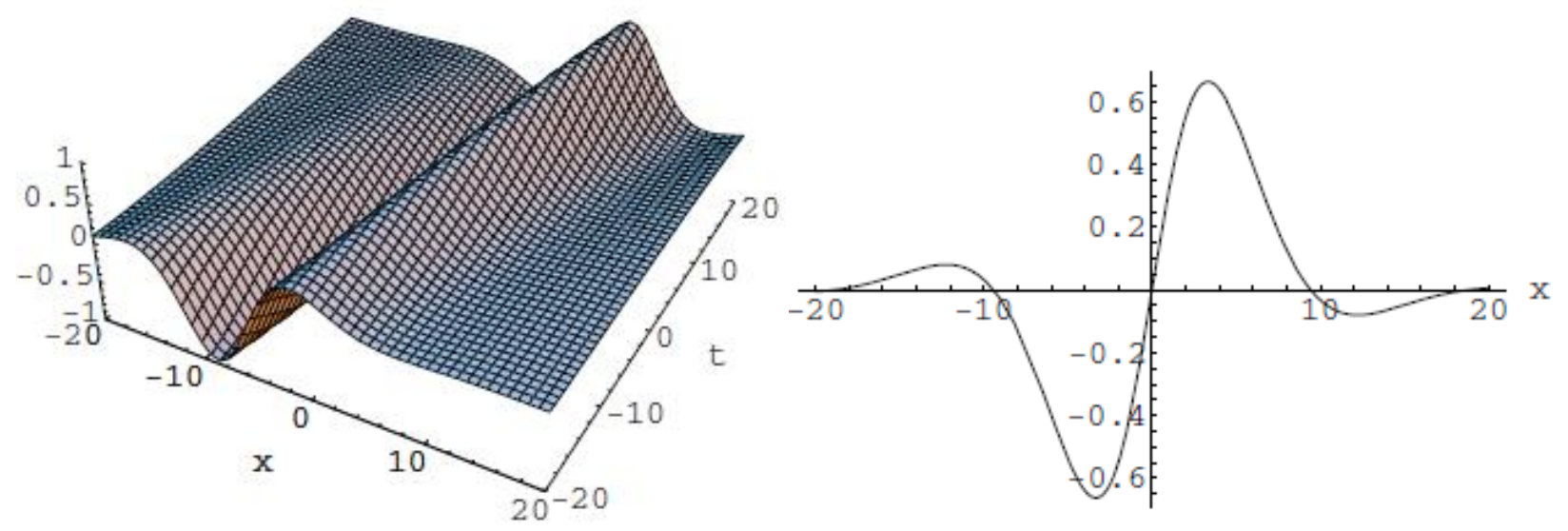

(c)
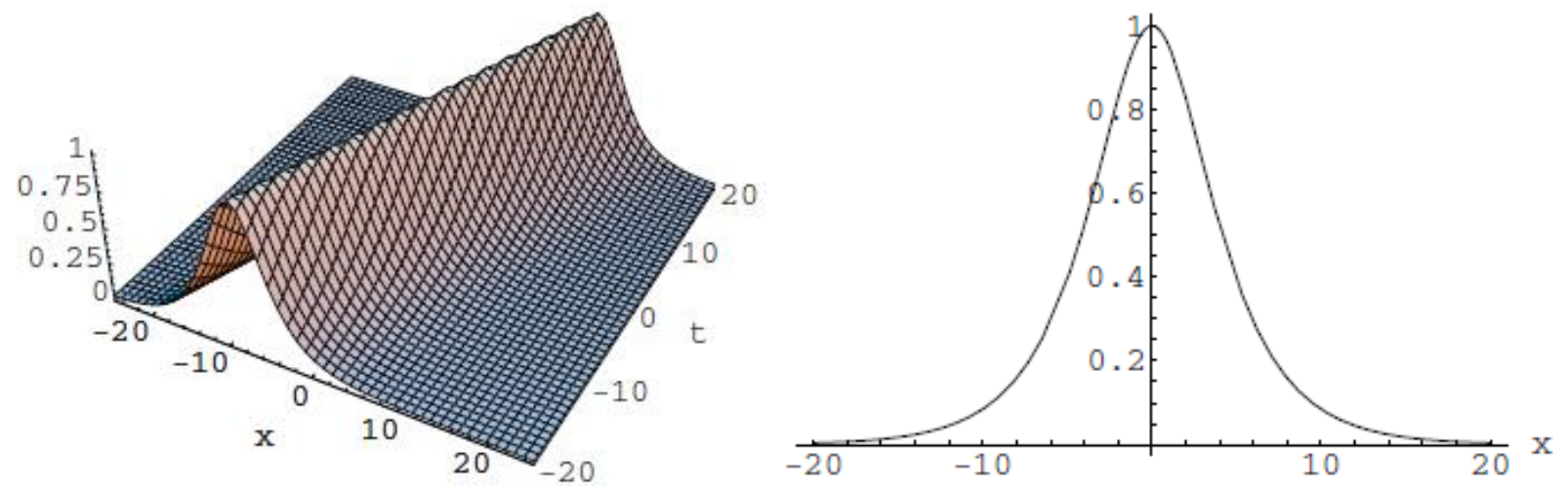

Figure 1. The travelling wave of solution and its position at $t=0$, where parameters $p=1$, $h=0.1 \quad(a)$ The real part $(b)$ the imaginary part $(c)$ the modulus

\section{Conclusion:-}

The traveling wave solutions $E_{2}, \eta_{2}, E_{3}, \eta_{3}, E_{4}, \eta_{4}$ and $E_{5}, \eta_{5}$ are completely new and have not found in earlier. But the solutions found in the Ref. [20] are the same to our obtain solutions $E(x, t)$ and $\eta(x, t)$ respectively. There are various types of traveling wave solutions that are particular interest in solitary wave theory. Many author implemented different methods to this system for obtaining the solutions. To best of our knowledge, the $\exp (-\Phi$ $(\xi)$ )-expansion method have not been implemented for constructing the traveling wave solutions of this model.

\section{References:-}

1. S.O. Rice, (1954) Mathematical analysis of random noise, in: N. Wax (Ed.), Noise and Stochastic Processes, Dover Publications, New York, pp. 133-294.

2. M.S. Longuet-Higgins, (1952) On the statistical distribution of the heights of sea waves, J. Marine Res. 11 1245-1266.

3. M.S. Longuet-Higgins, (1963) The effect of non-linearities on statistical distributions in the theory of sea waves, J. Fluid Mech. 17 459-480.

4. M.A. Tayfun, (1980) Narrow-band nonlinear sea waves, J. Geophys. Res. 85 1548-1552.

5. V.E. Zakharov. (1972). Collapse of Langmuir waves. Zh Eksp Teor Fiz, 62, 1745-1751.

6. Mv Golman. (1986). Langmuir wave solitons and spatial collapse in plasma physics. Physica, D 18, P. 67-76.

7. Michel Moisan, Jacques Pelletier. (2012): Hydrodynamic description of plasma, in: Physics of Collisional Plasmas, Springer. Netherlands.p. 203-335. 
8. L.H. Li. (1993). Langmuir turbulence equations with the self-generated magnetic field. Phys. Fluids, B 5, P. $350-356$.

9. B. Malomed, D. Anderson, M. Lisak, M.L. Quiroga-Teixeiro. (1977). Dynamics of solitary waves in the Zakharov model equations. Phys. Rev., E 55, P. 962-968.

10. J.H. He. (2005).Application of homotopy perturbation method to nonlinear wave equations, Chaos Solitons Fract., 26 (3), P. 695-700.

11. J.H. He, X.H. Wu. (2006). Construction of solitary solution and compaction-like solution by variational iteration method. Chaos Solitons Fract, 29 (1), P. 108-113.

12. J.H. He. (1999). Variational iteration method - a kind of nonlinear analytical technique: some examples. Int. J. Nonlinear Mech, 34 (4), P. 699-708.

13. J.H. He. (2000). Variational iteration method for autonomous ordinary differential systems. Appl. Math. Comput., 114 (2-3), P. 115-123.

14. Z.M. Odibat, S. Momani. (2006). Application of variational iteration method to nonlinear differential equations of fractional order. Int. J. Nonlinear Sci. Numer. Simul., 7 (1) P. 27-36.

15. Mehmet Giyas Sakar, Fevzi Erdogan, Ahmet Yildırım. (2012). Variational iteration method for the timefractional Fornberg- Whitham equation. Comput. Math. Appl., 63(9), P. 1382- 1388.

16. J.H. He, Guo-Cheng Wu, F. Austin. (2010). The variational iteration method which should be followed. Nonlinear Sci. Lett. A-Math. Phys. Mech., 1 (1), P. 1-30.

17. Arun Kumar. (2005). A variational method to study the Zakharov equation. Int. J. Math. Math. Sci., 22, P. 3703-3709.

18. D. Kaya, S.M. El-Sayed. (2004). A numerical simulation and explicit solutions of the generalized BurgerFisher equation. Appl. Math. Comput., 152, P. 403-413.

19. Pankaj, R. D. (2013) Laplace - Modified Decomposition Method to Study Solitary Wave Solutions of Coupled Nonlinear Klein-Gordon Schrödinger Equation, International Journal of Statistika and Mathematika, 5(1), pp 01-05

20. J.H. He. (2006). some asymptotic methods for strongly nonlinear equations. Int. J. Modern Phys., B 20, 10, P. 1141-1199.

21. E.M. Abulwafa, M.A. Abdou, A.A. Mahmoud. (2006). The solution of nonlinear coagulation problem with mass loss, Chaos Solitons Fract., 29 (2), P. 313-330.

22. Bekir. (2012). Multisoliton solutions to Cahn-Allen equation using double expfunction method. Phys. Wave Phenom., 20 (2), P. 118-121.

23. Abdul-Majid Wazwaz. (2008). 'tanh-coth and the sine-cosine methods for kinks, solitons, and periodic solutions for the Pochhammer-Chree equations'. Appl. Math. Comput, 195 (1), P. 24-33.

24. Kumar Arun, Pankaj R. D. (2015). Tanh-coth scheme for traveling wave solutions For Nonlinear Wave Interaction model. Journal of the Egyptian Mathematical Society, 23 (2), P. 282-285. 